Esta obra está bajo una Licencia Creative Commons Atribución-NoComercial-Compartirlgual 4.0 Internacional

(c) (1) (8) ()

El acotado uso de Facebook por los candidatos presidenciales en México 2018

Rafael Moreno Tostado y José Luis Arriaga Ornelas

DOI: https://doi.org/10.24215/16696581e216

\title{
El acotado uso de Facebook por los candidatos presidenciales en México 2018
}

\section{The limited use of Facebook by the presidential candidates in Mexico 2018}

\author{
Rafael Moreno Tostado rafaelmorenotostado@gmail.com \\ https://orcid.org/0000-0002-3952-9572 \\ José Luis Arriaga Ornelas llarriagao@gmail.com \\ http://orcid.org/0000-0002-0498-8461 \\ Universidad Autónoma del Estado de México (México)
}


Rafael Moreno Tostado y José Luis Arriaga Ornelas El acotado uso de Facebook por los candidatos presidenciales en México 2018

\section{Resumen}

Este artículo presenta los resultados de un ejercicio de seguimiento a la actividad que tuvieron en Facebook los tres principales candidatos a la presidencia de la República en la más reciente elección en México (2018). Se explora el tipo de interacción política que generaron y se cuestiona si el uso de las redes sociales está siendo acompañado por un cambio en el tipo de dinámicas comunicativas por parte de los partidos políticos en México. Los resultados se contrastan con el modelo de Mancini (1995), relacionado con las tres etapas por las que habría transitado la interacción política entre el poder político y los ciudadanos a lo largo del siglo XX. Los principales hallazgos son que el candidato ganador no fue el que más actividad tuvo en esta red social, que ninguno de los candidatos sostuvo una estrategia de interacción directa con sus contactos en esta red y que la interacción entre usuarios en los comentarios a las publicaciones de los candidatos fue la dinámica de comunicación política más relevante.

Palabras clave: comunicación política, Facebook, elecciones, candidatos, interacción

\section{Abstract}

This article presents the results of a follow-up exercise to the activity that the three main candidates for the presidency of the Republic had on Facebook in the most recent election in Mexico (2018). It explores the type of political interaction generated and asks if the use of social networks is being accompanied by a change in the type of communication dynamics by political parties in Mexico. The results are contrasted with the Mancini model (1995), related to the stages through which political communication had transited throughout the 20th century. The main findings are that the winning candidate was not the most active in this social network, that none of the candidates had a direct interaction with their contacts in this network and that the interaction between users was the dynamic of political communication more relevant.

Keeywords: political communication, Facebook, elections, candidates, interaction

\section{Introducción}

La política es, sobre todo, una actividad de comunicación (Valdés, 2015). Se desarrolla con base en procesos a través de los cuales se expresan, reciben y comprenden significados para generar un cierto clima de opinión colectiva, propicio para el ejercicio del poder. Como diría 
Rafael Moreno Tostado y José Luis Arriaga Ornelas El acotado uso de Facebook por los candidatos presidenciales en México 2018

Weber (1979), estos procesos se encaminan a crear las condiciones necesarias para el funcionamiento de cualquier relación dominante-dominado. El hecho de que durante el siglo XX la comunicación a través de medios masivos se haya convertido en la forma esencial de contacto entre los seres humanos (Reyes, 2007), propició la aparición de una área de estudios interesada por el modo en que se presentan las relaciones entre los medios de comunicación y la opinión pública: la "comunicación política". Es un nombre que empezó a ocuparse hacia mediados del siglo pasado en los Estados Unidos (Eulau, Eldersveld y Janowitz, 1956) y en el que se incluyeron los estudios pioneros sobre persuasión e influencia de los medios masivos, propaganda, campañas políticas, entre otros, realizados por gente como Pakard, Lazarfeld, Berelson y Gaudet y algunos más.

A medida que este tipo de estudios fueron avanzando, los datos empezaron a evidenciar la relatividad de lo que en un primer momento se asumía como poder absoluto de los medios para manipular a los individuos (Guerrero, 2003). Por esta razón, ya para finales de los años "sesenta, había trabajos importantes (...) como los de Kurt Lang y G. Lang, Valdimer Key, Jay Blumer y James Halloran. Algunos defendían que los individuos practican una exposición selectiva de la información que reciben de los medios, mediante ésta seleccionan y discriminan mensajes con base en creencias y valores previamente determinados" (Reyes, 2007, p. 89).

Luego, los estudios de comunicación política enfocaron su interés en el comportamiento del electorado, en el contenido del discurso, en la propaganda, el marketing y el debate político, entre otros temas; hasta que devinieron en "un campo interdisciplinar de estudios, utilizando conceptos desde la comunicación, desde la ciencia política, desde el periodismo, la sociología, la psicología, la historia, la retórica, y otros campos" (Kaid, 2004, p. XIII). Quizá sea Wolton quien lo resume mejor:

El rótulo "Comunicación Política" se refería en principio al estudio de la comunicación del gobierno hacia el electorado, después designó la circulación de los mensajes de los políticos especialmente en las campañas electorales- y más tarde el campo se fue extendiendo, hasta incluir el rol de los medios en la formación de la opinión pública y la influencia de los sondeos en la política. Luego el rótulo incluyó el estudio del rol de la comunicación en la vida política en el sentido más amplio, incluyendo los medios, los sondeos, el marketing político y la publicidad, especialmente en los períodos electorales (Wolton, 1990, p. 10).

El presente trabajo, asumiendo ese proceso de expansión que aún presenta el campo de la comunicación política, su interdisciplinariedad y su evolución relacionada con los nuevos problemas en materia de comunicación y actividades políticas y de gobierno, lo que hace es ofrecer resultados de una investigación realizada en las pasadas elecciones presidenciales en 
México (celebradas el 1 de julio de 2018) en la cual el principal interés radicó en documentar el comportamiento que tuvieron en Facebook los tres principales candidatos.

Explorar el uso de las nuevas tecnologías digitales (y especialmente las redes sociales) en las actividades políticas no es algo nuevo. Se ha enfocado, principalmente, su utilización exitosa en campañas electorales, ahora también llamadas "cibercampañas" (Gutiérrez, Islas y López, 2000), en las que ha destacado, por ejemplo, el uso de herramientas como twitter (Rodríguez y Ureña, 2012). Por su trascendencia, también fue materia de interés el caso de Barack Obama en su campaña de 2008 (Delany, 2009; Peytibi, Rodríguez y Gutiérrez, 2008; Hendricks y Denton, 2010), así como lo ocurrido en otros países en los que las posibilidades de la Web 2.0 abren nuevos derroteros para las campañas electorales (Túñez y Sixto, 2011; Kreiss, 2015; Dader, 2016). Y, desde luego, también ya se han realizado estudios comparativos entre países en los que el uso de las redes sociales ha sido destacado (Cárdenas, Ballesteros y Jara, 2017). Para el caso que ocupa a este trabajo, la interrogante central fue: ¿el uso de las redes sociales, ampliamente extendido entre la población mexicana, está siendo acompañado por un cambio en el tipo de dinámicas comunicativas por parte de los partidos políticos en México? Para encontrar respuestas se buscó describir el comportamiento que los candidatos presidenciales tuvieron en la red social Facebook durante la campaña presidencial de 2018, sobre todo buscando identificar las formas de interacción entre ellos y los potenciales electores, dadas las posibilidades que la propia plataforma permite. Los datos descriptivos que se obtuvieran serían analizados para abonar a la discusión sobre la vigencia de la propuesta que hizo Mancini (1995) al respecto de las tres etapas por las que habría transitado la interacción política entre el poder político y los ciudadanos a lo largo del siglo XX: 1) fase de la comunicación del aparato, 2) fase pionera de la comunicación de masas y 3) fase del poder de los medios.

El proceso electoral dentro del cual se desarrolló la campaña presidencial aludida fue el más grande en la historia de México (Padilla, 2018), no sólo por el número de votantes que podrían participar, sino por la cantidad de cargos en juego, que rebasaban los 3,000. Además, el ganador de la Presidencia de la República obtuvo más votos de los que obtuvo jamás otro Presidente electo: 30,110,327 votos (Tribunal Electoral, 2018). Estos factores se suman a otros para hacer más interesante el caso de la elección de 2018 en términos de comunicación política: primero, el hecho de que en el año 2000 el Partido Revolucionario Institucional (PRI), que había gobernado por varias décadas y se había caracterizado por implementar un modelo de comunicación política fuertemente controlado por el Estado (Reyes, 2015), perdió la presidencia a manos del Partido Acción Nacional (PAN); segundo, en el año 2006 se presentó la contienda más cerrada de la historia del país (menos de un punto porcentual separó al ganador el segundo lugar), con un signo distintivo: campañas intensas de spots en radio y la 
Rafael Moreno Tostado y José Luis Arriaga Ornelas El acotado uso de Facebook por los candidatos presidenciales en México 2018

televisión (Juárez, 2007) para denostar al candidato que terminó perdiendo por escasos 200 mil votos; y, tercero, el nuevo relevo entre PAN y PRI cuando en el año 2012 el segundo regresa a la Presidencia de la República en el marco de una gran exposición televisiva de su candidato, Enrique Peña Nieto (Valdés, 2015).

Por lo que hace a las razones de poner atención a la propuesta de Mancini (1995) es necesario señalar la adecuación que su tipología logra al contrastarse con las etapas del México postrevolucionario en materia de comunicación política, pero permite preguntarse por la vigencia o el potencial surgimiento de una nueva etapa. Dicho autor sugiere tres etapas: la primera ("comunicación del aparato") se caracteriza -afirma- por un tipo de comunicación que pasaba por el contacto personal asegurado por los grandes aparatos de organización de los partidos. Y en el caso de México, el PRI, "heredero directo de la Revolución Mexicana y monopolista de su ideología, criatura estatal, partido corporativo" (Báez, 2002, p. 234), nació y se consolidó como "un partido de corporaciones, en el que sus unidades de base eran las organizaciones, mientras que los individuos resultaban elementos secundarios. Eran las organizaciones (o el pueblo organizado) las que constituían el Partido" (Córdova, 1989, p. 188). Así que el tipo de comunicación política que le sostenía, sobre todo en sus primeras décadas, reúne las características de la mencionada primera etapa.

La segunda etapa sugerida por Mancini ("fase pionera de la comunicación de masas") estaría caracterizada por una relación subalterna entre los medios de comunicación y los aparatos de partido. Y, en el caso Mexicano, se ha documentado (Guerrero, 2010; Trejo, 1992; Zarur, 1996; Mejía, 1989; Fernández, 1996) que el Estado emergido de la Revolución hizo lo necesario para establecer con los medios de comunicación masiva una relación de tipo corporativo y/o de disciplina y lealtad. Lo hizo otorgando diversos beneficios técnicos y económicos que favorecieron el desarrollo de negocios rentables, pero siempre a cambio del apoyo irrestricto al régimen político, lo cual aseguró mediante un marco normativo y mecanismos informales que le aseguraban los apoyos y subordinación tanto de la prensa escrita como de la radio y la televisión. Esta etapa se prolongaría hasta el último tramo del siglo XX.

Y en relación con la tercera etapa ("fase del poder de los medios"), en México la misma se habría estrenado con el nuevo siglo, cuando el Estado dejó de tener la propiedad y/o el dominio que ejerció sobre los medios durante casi todo el siglo XX (Trejo, 2001), cuando las elecciones se convirtieron en verdaderas competencias (Aceves, 2005) y en donde el marketing político, sus estrategias y técnicas se han ido incorporando a los procesos electorales (Kuschick, 2009), hasta llegar a esquemas de franco poder mediático, puesto al servicio del mejor postor, donde ellos (los medios) se sabían centrales en la contienda para colocar candidatos de cara a los comicios (Dafonte y Martínez, 2016), llegando incluso al punto en que pareciera que "los 
medios de comunicación son ya subsistemas que interactúan autónomamente entre sí, haciéndose de una imagen social y actuando con plena autonomía (lo cual) debilita la figura de los partidos políticos" (Vega, 2003, p. 5).

El poder que se presume tienen los medios para posicionar a un candidato se basaría, sobre todo, en campañas de spots en radio y televisión. En México, la ley prohíbe a los partidos políticos y candidatos contratar spots y es el Instituto Nacional Electoral (INE) el que lo hace y reparte los mismos. Para la elección de 2018, fueron asignadas pautas para 26 millones de spots (utilizables para las distintas campañas que estaban en curso que eran varias, incluyendo Presidente de la República, Diputados Federales y Senadores). La repartición que realizó el INE es muy interesante a la luz de los resultados finales, pues el candidato Andrés Manuel López Obrador, quien resultó ganador, era el que menos spots pudo utilizar durante su campaña (2.4 millones de spots en 90 días), en tanto, el candidato Ricardo Anaya Cortés que era el que disponía de más spots (6.5 millones) quedó en un lejano segundo lugar. Y el candidato José Antonio Meade Kuribreña, aún teniendo la segunda mayor cantidad de spots (5.9 millones) quedó casi a 30 puntos porcentuales del ganador de la contienda. Esto desde luego que aporta razones para preguntarse por la vigencia de la tercera etapa a la que se refiere Mancini (1995) y provoca cuestionarse sobre el rol jugado por las nuevas herramientas de comunicación política, como son las redes sociales virtuales.

\section{La elección del 2018 y la metodología a seguir}

Para los fines ya anteriormente expuestos, se realizó un registro cuantitativo sobre la actividad de las cuentas oficiales de Facebook de los tres candidatos con posibilidades reales de ganar la contienda electoral del 2 de julio de 2018. Se contabilizaron los principales indicadores de la actividad digital en Facebook de Andrés Manuel López Obrador (Candidato de la coalición "Juntos haremos historia" Morena), Ricardo Anaya Cortés (candidato la coalición "Por México al frente") y José Antonio Meade Kuribreña (Candidato de la coalición "Todos por México"). El seguimiento se dividió en cuatro momentos de relevancia durante las campañas, con el fin de obtener datos representativos de los momentos de mayor exposición mediática de los candidatos a la Presidencia.

\section{Primer momento:}

Inicio Oficial de Campañas Electorales para la Presidencia de la República.

\section{Segundo momento:}

Primer Debate Presidencial en la Ciudad de México.

Question, Vol. 1, N. ${ }^{\circ}$ 64, octubre-diciembre 2019. ISSN 1669-6581

Instituto de Investigaciones en Comunicación | Facultad de Periodismo y Comunicación Social | Universidad Nacional de La Plata 
Rafael Moreno Tostado y José Luis Arriaga Ornelas El acotado uso de Facebook por los candidatos presidenciales en México 2018

\section{Tercer momento:}

- $\quad$ Segundo Debate Presidencial en la ciudad de Tijuana.

\section{Cuarto momento:}

- $\quad$ Tercer Debate Presidencial en la ciudad de Mérida.

Posteriormente se levantó un registro del comportamiento de las cuentas oficiales de los candidatos en cuanto al número de seguidores, al igual que del comportamiento que tuvieron éstas con base en el número de publicaciones ejecutadas, cantidad de reacciones obtenidas y el total de veces que se compartieron por los seguidores de los políticos. Después del registro se buscaría ensayar una tipificación de las publicaciones realizadas por los candidatos en los cuatro momentos de relevancia vividos en la campaña, haciendo énfasis en el tipo de información y comunicación de las divulgaciones que los políticos realizaron en Facebook. El propósito de tal tipificación sería identificar el modelo comunicativo institucional utilizado por los partidos políticos y su adaptación a las nuevas tecnologías informáticas. Recuérdese que animaba a la investigación el saber si los candidatos establecen comunicación con los usuarios, seguidores o "amigos"; explorar si los políticos fomentan el diálogo o detonan confrontaciones; averiguar si sólo informan e indagar si en la plataforma se utilizan los mecanismos que dan apertura al diálogo político o únicamente al diálogo entre usuarios. El Registro cuantitativo de redes sociales que se efectuó utilizó la siguiente simbología:

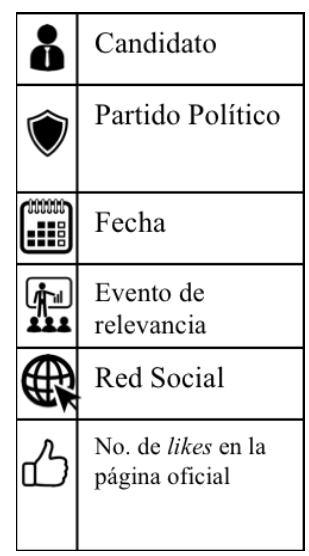

\begin{tabular}{|c|c|}
\hline iٔ & $\begin{array}{l}\text { No. de followers en la } \\
\text { página oficial }\end{array}$ \\
\hline 틀 & $\begin{array}{l}\text { Cantidad de } \\
\text { publicaciones }\end{array}$ \\
\hline 606 & $\begin{array}{l}\text { No. de reacciones } \\
\text { en total }\end{array}$ \\
\hline & $\begin{array}{l}\text { No. de comentarios } \\
\text { en total }\end{array}$ \\
\hline 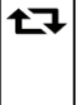 & $\begin{array}{l}\text { No. de veces que se } \\
\text { compartieron las } \\
\text { publicaciones del } \\
\text { día }\end{array}$ \\
\hline
\end{tabular}

Primer momento: El día 30 de marzo de 2018 dieron inicio de manera oficial las campañas electorales para la Presidencia de la República y con esto se llevó a cabo la recopilación de los primeros datos sobre las cuentas de Facebook de los candidatos. Para este primer momento 
se destaca la inexistente actividad digital del candidato Andrés Manuel López Obrador. Igualmente debe destacarse que el candidato que generó más reacciones, comentarios e interacciones, fue el abanderado de la coalición "Por México al Frente", Ricardo Anaya, quien ese día de inicio de campaña hizo cinco publicaciones en su perfil de Facebook.

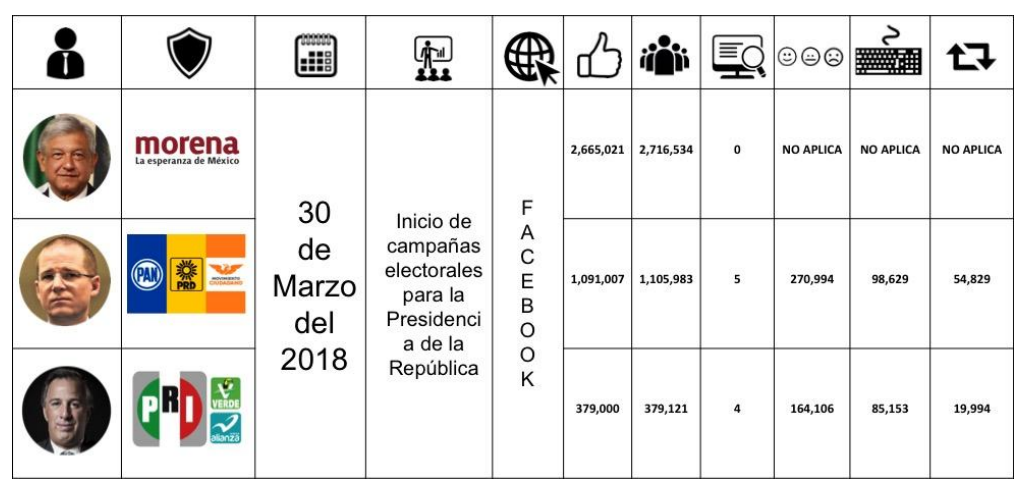

Tabla 1. Registros hechos el día de inicio oficial de campañas electorales para la Presidencia de la República.

Segundo momento: El crecimiento orgánico de cada una de las cuentas oficiales se desarrolló de la siguiente manera:

- El candidato Ricardo Anaya Cortés ganó 293,658 "Me gusta" y 304,083 nuevos seguidores del 30 de marzo al 22 de abril, fecha del Primer Debate Presidencial.

- Andrés Manuel López Obrador obtuvo 150,358 nuevos "Me gusta" en su página oficial de Facebook y 164,508 seguidores en el mismo periodo de tiempo.

- El abanderado de la coalición "Todos por México", José Antonio Meade, sumó 96,304 "Me gusta" y 111,102 nuevos seguidores en esta primera etapa.

A estas alturas de la campaña el candidato Ricardo Anaya fue quien más veces utilizó la red social para hacer un total de catorce publicaciones, seguido de José Antonio Meade, quien ejecutó cuatro publicaciones y, por último, el abanderado de Morena publicó en una sola ocasión. 


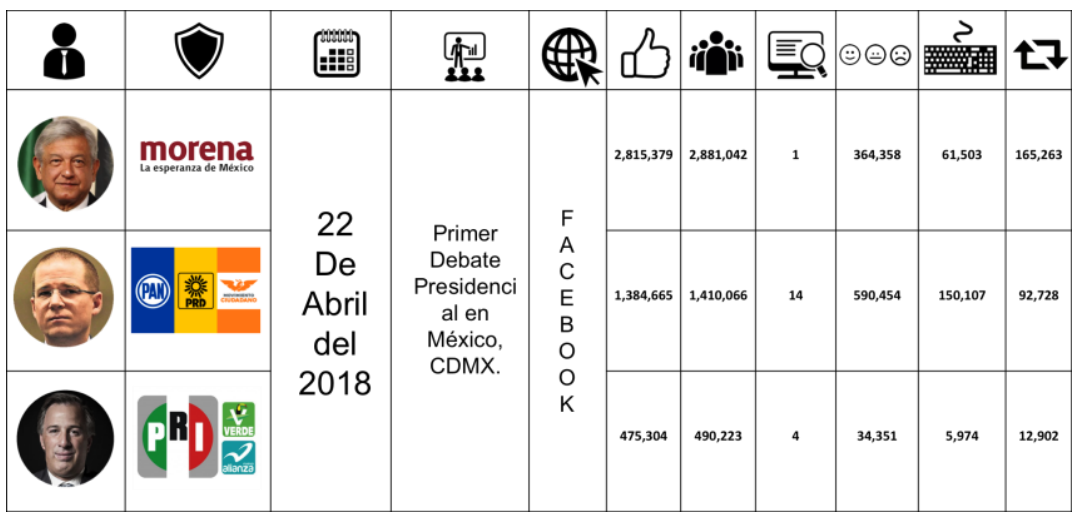

Tabla 2. Obtenido el día del Primer Debate Presidencial en la Ciudad de México.

Tercer momento: Se corresponde con la realización de un segundo debate oficial, que se llevó a cabo el día 20 de mayo de 2018, en la ciudad de Tijuana. Para este momento los datos analizados arrojan que: el candidato de la coalición "Por México al Frente", Ricardo Anaya Cortés, continuó puntero en la suma de nuevos "Me gusta" y seguidores, durante el periodo del 22 de abril al 20 de mayo, sumando un total de 402,029 likes y 408,145 followers.

Por su parte, Andrés Manuel López Obrador se colocó en el segundo puesto en cuanto al crecimiento total de su página oficial, sumando un total de 375,544 "Me gusta" y 422,923 nuevos seguidores. Mientras que José Antonio Meade ganó en este mismo periodo de tiempo un total de 102,250 "Me gusta" y 105,361 seguidores. Asimismo, en este segundo debate, llama la atención que el candidato de la coalición "Juntos Haremos Historia no realizó de nueva cuenta ningún tipo de publicación en la red social con motivo de este importante momento de la campaña.

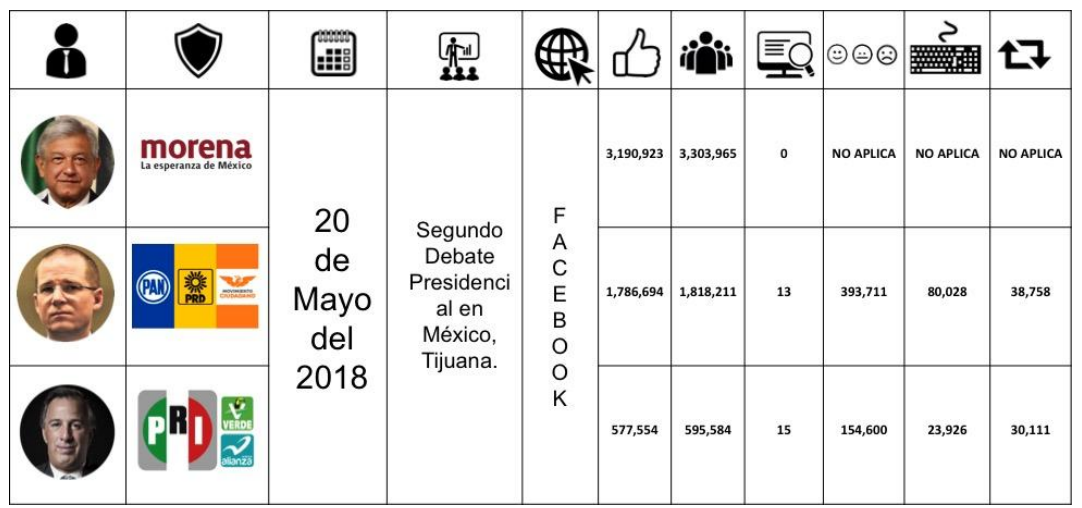

Tabla 3. Obtenido el día del Segundo Debate Presidencial en la ciudad de Tijuana, México. 
Cuarto momento: Los tres candidatos con posibilidades reales de ganar la contienda electoral llegaron con números mucho más sólidos a esta etapa de la campaña. En primer lugar, López Obrador concluyó puntero en cuanto a número de "Me gusta" y seguidores, sumando para este tercer debate un total de 3,367,516 likes y 3,489,190 followers. Ricardo Anaya, por su parte, logró llegar a los 2,024,941 "Me gusta" y sumó un total de 2,061,595 seguidores. Y José Antonio Meade no logró sumar ni un millón de simpatizantes, pues únicamente pudo obtener 727,817 likes y 753,121 seguidores.

Llama la atención que, para este tercer y último Debate Presidencial, el candidato Meade Kuribreña realizó un total de 21 publicaciones y, aunque fue el candidato que más veces posteó en su perfil de Facebook, obtuvo el menor número de reacciones, comentarios y fue compartido en un menor número de veces, a diferencia de Ricardo Anaya, quien realizó el mayor número de publicaciones ejecutadas a lo largo de su gestión en este cuarto momento, un total de quince. Finalmente, fiel a su estrategia, Andrés Manuel posteó en una única ocasión. Con esta publicación el candidato de Morena se posicionó muy cerca de Meade Kuribreña en cuanto a número de reacciones, comentarios y compartidos, hablándonos de la eficiencia de sus publicaciones.

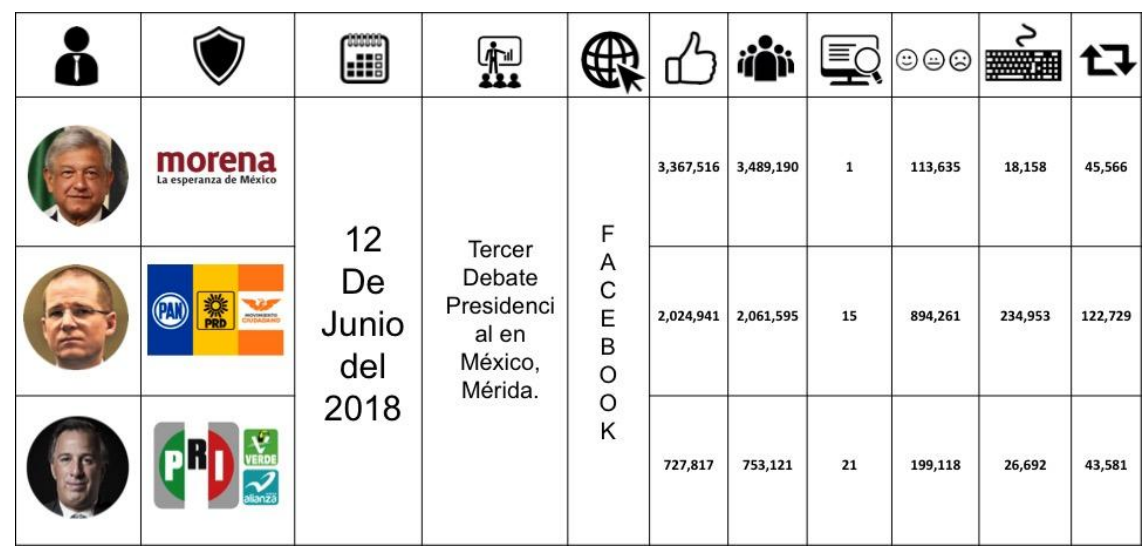

Tabla 4. Obtenido el día del Tercer Debate Presidencial en la ciudad de Mérida, México.

Como se dijo antes, luego de este registro cuantitativo se realizó una tipificación de las publicaciones emitidas por los tres candidatos a la Presidencia de la República, con la finalidad de averiguar si los políticos utilizaron la red social con la intención de fomentar el diálogo, detonar confrontaciones o por el simple hecho de informar a sus simpatizantes "algo" relacionado con el desarrollo de su campaña en los momentos de mayor exposición mediática. 
Rafael Moreno Tostado y José Luis Arriaga Ornelas El acotado uso de Facebook por los candidatos presidenciales en México 2018

A continuación, se presentan ejemplos de los tipos de acciones identificados: comunicativas, informativas e informativas de contraste.

\section{Comunicativa}

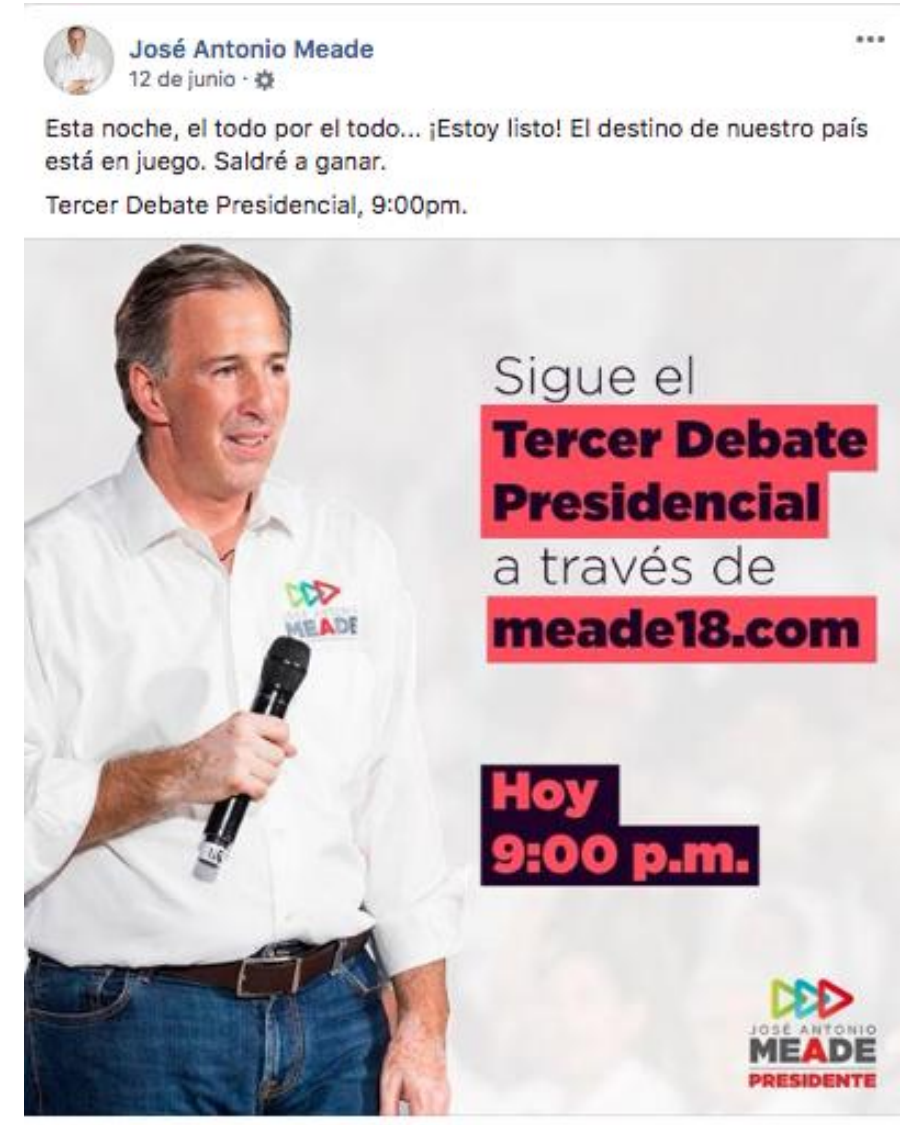

Ejemplo 1. Fuente: Facebook. Página Oficial de José Antonio Meade. 12 de junio del 2018.

A pesar de que la palabra comunicación tiene múltiples acepciones, preferimos subrayar aquélla que indica que el acto de comunicarse es ponerse en contacto con otra persona (o con otras) y lograr un intercambio en el ánimo de transmitir algo, de informarse de algo, de construir algo o de esperar una acción. En esta publicación, realizada por el candidato José Antonio Meade, el día del Tercer Debate Presidencial, se identifica uno de los pocos gestos 
Rafael Moreno Tostado y José Luis Arriaga Ornelas El acotado uso de Facebook por los candidatos presidenciales en México 2018

comunicativos hechos en la campaña a través de su cuenta de Facebook, pues hacía una invitación a la acción: concretamente, ver el debate. Esta divulgación contiene los ingredientes necesarios para tipificarla como una publicación comunicativa, ya que el político lanza la invitación esperando una reacción de sus simpatizantes. La comunicación va orientada a que los usuarios hagan algo.

\section{Informativa}

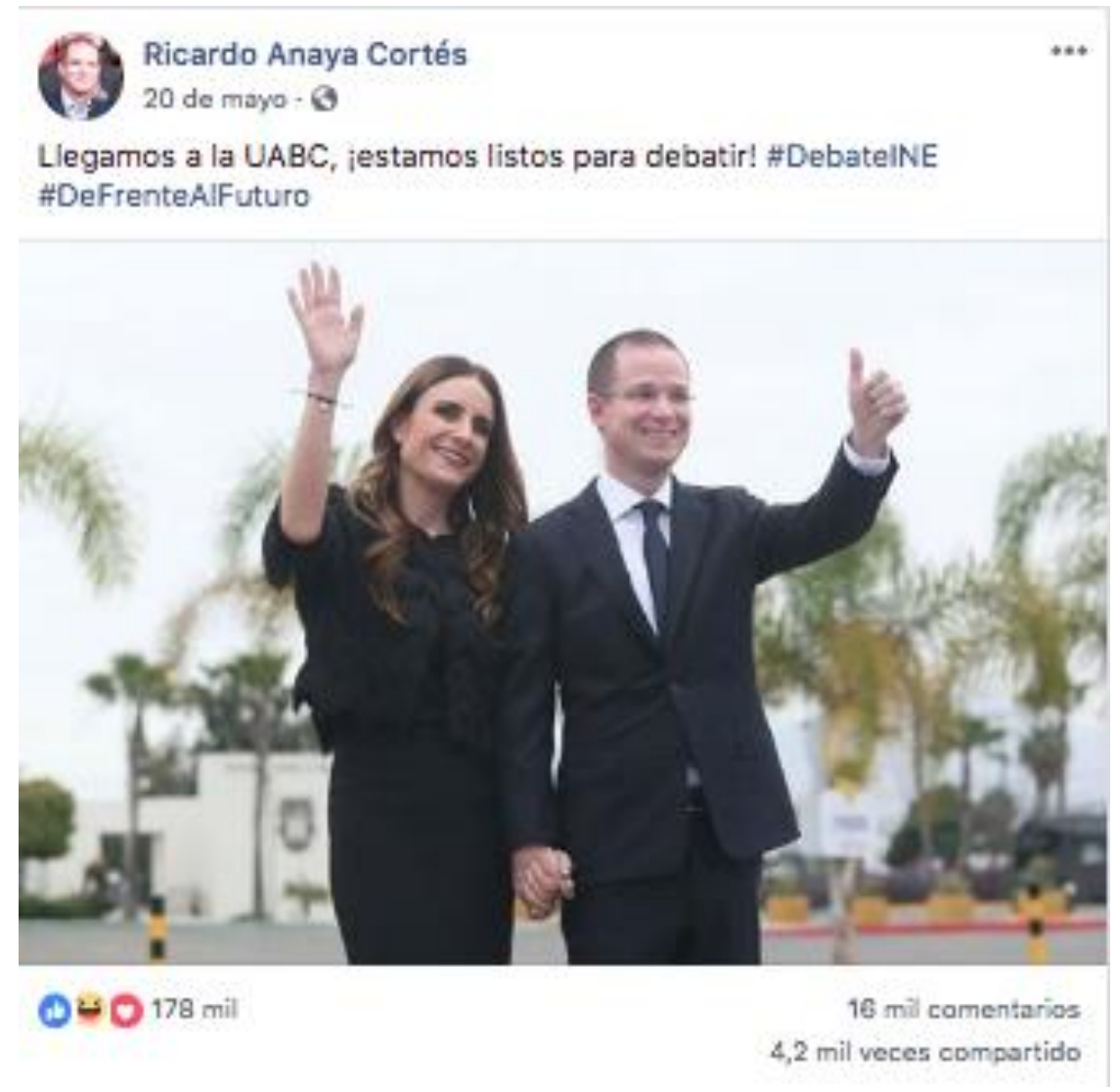

Ejemplo 2. Fuente: Facebook. Página Oficial de Ricardo Anaya Cortés. 20 de mayo del 2018.

El otro tipo de publicaciones que se pudo identificar entre las realizadas por los contendientes a la Presidencia de la República en sus cuentas de la red social bajo análisis fueron las divulgaciones informativas. Este tipo de publicaciones son unidireccionales, no esperan respuesta, sólo proporcionan un dato o asignan sentido a una acción. Se sabe que un mitin político se permite arengar, pedir el voto para el partido ante una audiencia cautiva de partidarios y seguidores, pero en las redes sociales los políticos divulgan ante propios y 
Rafael Moreno Tostado y José Luis Arriaga Ornelas El acotado uso de Facebook por los candidatos presidenciales en México 2018

extraños: ante los extraños, la labor política sería convencerlos y cautivarlos, pero con este tipo de publicaciones no se logra nada, no se comunica, se informa únicamente.

\section{Informativa de Contraste}

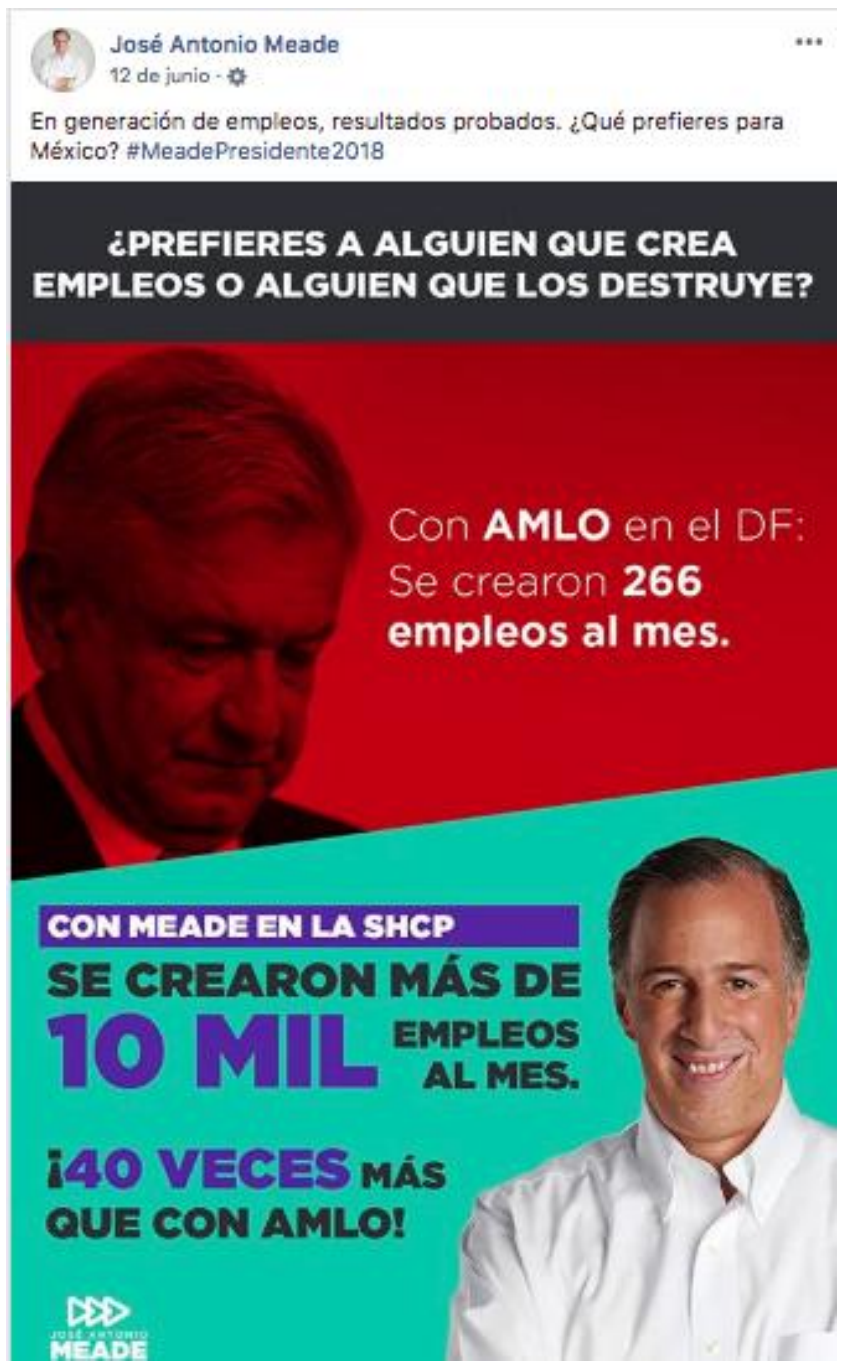

Ejemplo 3. Fuente: Facebook. Página Oficial de José Antonio Meade. 12 de junio del 2018.

Las publicaciones de contraste son otro tipo de divulgaciones que los candidatos realizaron durante sus campañas. En estos casos, como su nombre lo indica, se generan dos informaciones articuladas que generan un efecto de contraste al compararlas directamente. En este caso, el representante de la coalición "Todos por México" informa los resultados que ha obtenido previamente en su trayectoria política, en comparación con su rival, Andrés Manuel 
López Obrador, para que al contrastar ambos datos resulte evidente su capacidad para la creación de empleos. Este tipo de publicaciones tienen la finalidad de hacer evidente el sentido por el cual el candidato se maneja, los polos de sus propuestas y la dirección de su campaña, para así diferenciarse de sus contrincantes.

Ahora bien, después de las acciones primigenias emprendidas por los candidatos políticos 0 sus equipos de campaña, es importante considerar las reacciones que se tuvieron como respuestas por parte de sus contactos o seguidores en la red social. A continuación se presentan algunas de las acciones realizadas por los usuarios de Facebook en respuesta a la acción primaria de los candidatos. En este apartado se muestra la importancia, el volumen y el poder que tiene el usuario de la red social ante los mensajes recibidos.

Ejemplo:

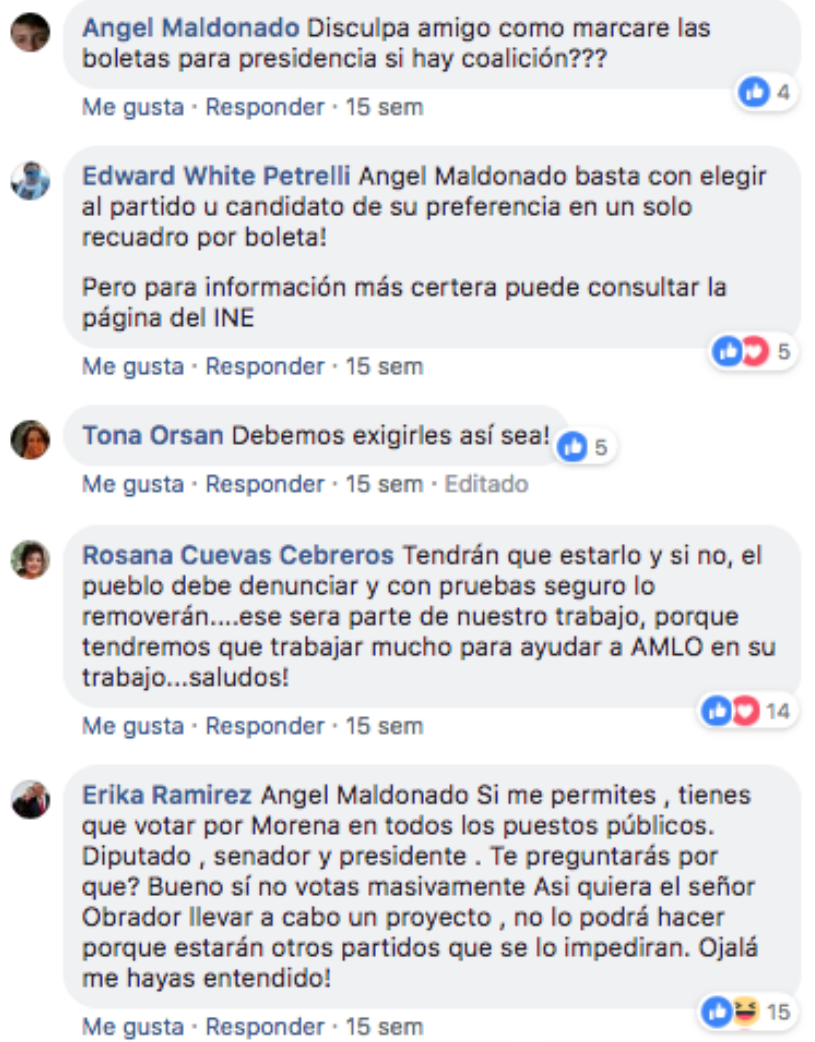

Ejemplo 4. Fuente: Facebook. Página Oficial de Andrés Manuel López Obrador. 22 de abril del 2018. 
En el ejemplo anterior es posible notar la importancia que tienen los usuarios en la red social: tras el cuestionamiento de un usuario se esperaría la réplica por parte del candidato, pero más bien su duda dio a los usuarios la oportunidad de comunicar de manera precisa los pasos a seguir para ejercer su voto de manera correcta. También podemos notar la interacción con otros usuarios de la red social al intentar dar respuesta a un planteamiento de relevancia.

El volumen de los usuarios involucrados se puede evidenciar en el siguiente ejemplo; en esta publicación realizada por José Antonio Meade, el día 30 de marzo, se observa que la divulgación recolectó un total de 42 mil reacciones; enseguida, un comentario obtuvo un total de 4,9 mil reacciones y 340 respuestas realizadas por otros usuarios; y, por debajo de este comentario, encontramos un comentario nuevo, con 2,2 mil reacciones y 54 respuestas. En la parte inferior derecha encontramos que estos son únicamente dos comentarios de 22,280 más que hubo; si sumamos la cantidad de reacciones que se recolectaron a partir de los comentarios en esta publicación, resulta evidente que el volumen y la actividad de los usuarios son lo que anima la actividad en la red social más que las acciones del candidato que realizó tal publicación primaria. 


\begin{tabular}{|c|c|}
\hline $\begin{array}{l}\text { Resolveré tus problemas desde el primer } \\
\text { día }\end{array}$ & Más información \\
\hline \multirow[t]{3}{*}{ Cᄇㅜ $42 \mathrm{mil}$} & \multirow{3}{*}{$\begin{array}{l}22 \text { mil comentarios } \\
5,7 \text { mil veces compartido } \\
1,3 \text { mill. reproducciones }\end{array}$} \\
\hline & \\
\hline & \\
\hline D Me gusta & Compartir \\
\hline
\end{tabular}

Todos los comentarios

Escribe un comentario...

Valeria Ramirez Me da tanta vergũenza ver como hay personas que apoyan a este señor, les hace falta conocer tanto, además 20 años en el servicio público y no ha dejado ningún avance en ninguna instancia en la que ha estado, de verdad vergonzoso y preocupante

Me gusta $\cdot$ Responder $\cdot 20$ sem

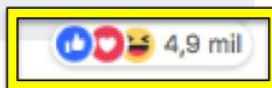

$\rightarrow 340$ respuestas

Victor Mendez Si el señor tiene mucha experiencia para poner más impuestos más gazolinazo más corrupción más pobreza más inseguridad más desaparecidos más violencia México eso quieres para tus hijos despierta hagamos un cambio verdadero ni un voto más al PRI.

Me gusta - Responder - 20 sem

(1) $9 \div 2,2 \mathrm{mil}$

454 respuestas

Ver más comentarios

Ejemplo 5. Fuente: Facebook. Página Oficial de José Antonio Meade. 30 de marzo del 2018.

Cabe señalar que el único candidato que en ocasiones intentó dar la apariencia de interacción fue Ricardo Anaya Cortés, quien, en algunos comentarios de sus publicaciones, realizó respuestas, bien armadas, a algunos de los comentarios que se generaban por parte de los usuarios. Lo curioso del caso es que encontramos estas respuestas en comentarios únicamente positivos y en apoyo al candidato y al partido, pero ninguno haciendo frente a los planteamientos críticos por parte de los usuarios. 
Rafael Moreno Tostado y José Luis Arriaga Ornelas El acotado uso de Facebook por los candidatos presidenciales en México 2018

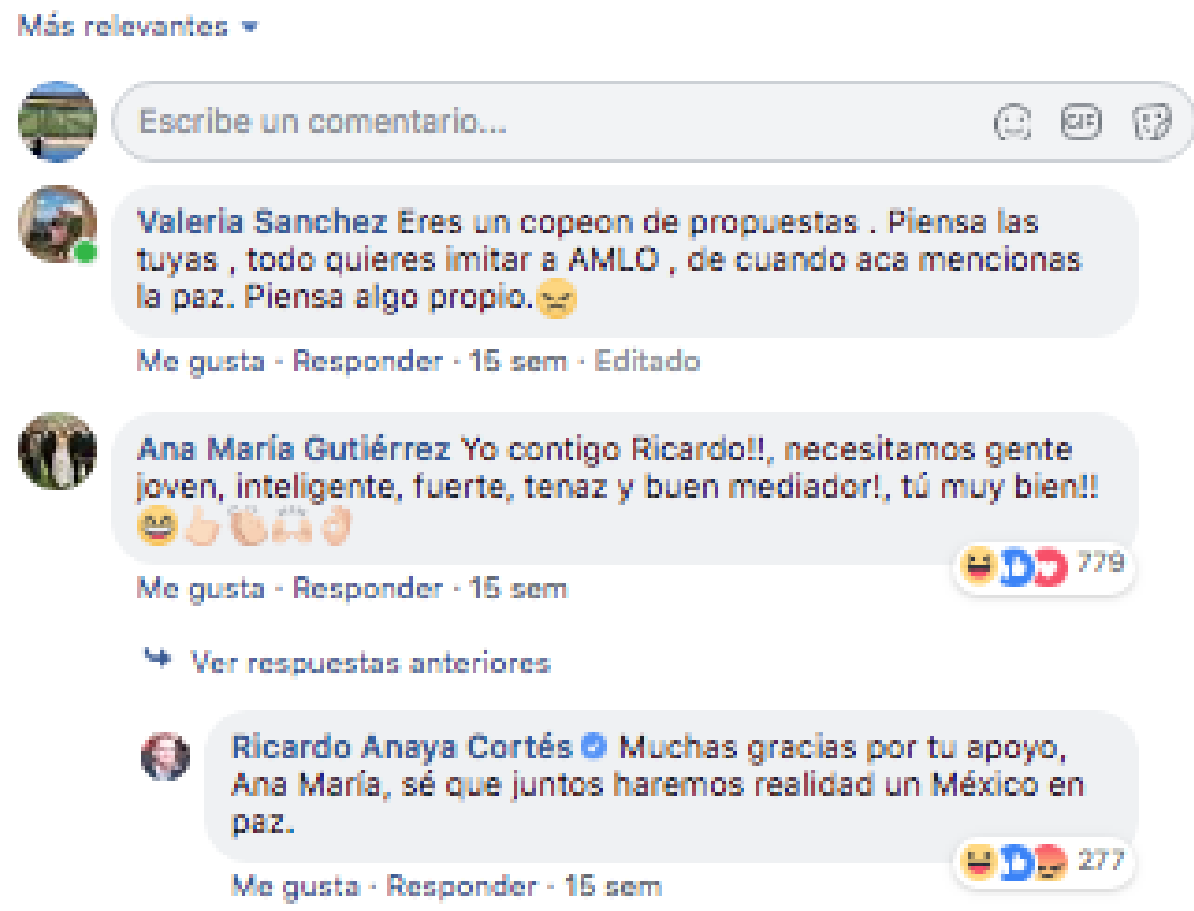

Ejemplo 6. Fuente: Facebook. Página Oficial de Ricardo Anaya Cortés. 12 de junio del 2018.

A continuación se presenta el concentrado de la cantidad de publicaciones ejecutadas por los candidatos en los cuatro momentos estudiados de la campaña electoral y su aplicación a los tres tipos de publicaciones identificados.

\begin{tabular}{|l|l|l|l|l|}
\hline Total & $\begin{array}{l}\text { Total } \\
\text { publicaciones de } \\
\text { emitidas en los } \\
\text { momentos } \\
\text { estudiados de la } \\
\text { campaña }\end{array}$ & $\begin{array}{l}\text { Acción } \\
\text { Comunicativa }\end{array}$ & Informativa & $\begin{array}{c}\text { Informativa } \\
\text { de Contraste }\end{array}$ \\
\hline AMLO & 2 & 0 & 2 & 0 \\
\hline
\end{tabular}




\begin{tabular}{|l|l|l|l|l|}
\hline $\begin{array}{l}\text { Ricardo } \\
\text { Anaya }\end{array}$ & 47 & 7 & 37 & 3 \\
\hline $\begin{array}{l}\text { José A. } \\
\text { Meade }\end{array}$ & 44 & 5 & 32 & 7 \\
\hline
\end{tabular}

\section{Discusión}

Los datos obtenidos sobre la actividad en Facebook por parte de los candidatos permite inferir que ellos y sus equipos de campaña de la contienda electoral 2018 no contaron entre sus estrategias prioritarias con una directiva planificada para el uso eficiente de esta red social. Lo más evidente es que el candidato que resultó ganador, Andres Manuel López Obrador, hizo un uso muy acotado de su perfil de Facebook. En tanto, conforme avanzaba la campaña, fue notorio que los candidatos Ricardo Anaya Cortés y José Antonio Meade Kuribreña incrementaron sus acciones en sus respectivos perfiles, llegando a efectuar decenas de publicaciones. El primero podría haberlo hecho como parte de su esfuerzo uno por alcanzar al puntero, en tanto que el segundo buscando incrementar los números de su cuenta, que simplemente nunca logró despegar. A Ricardo Anaya, el candidato que más veces publicó en la plataforma, podrían darle la razón los números (pues fue quien logró un mayor número de reacciones, comentarios y réplicas por parte de sus seguidores) aunque el tipo de crecimiento que experimento podría estar relacionado con el uso de bots, con la intención fortalecer artificialmente sus números, sintiéndose cercano al candidato que finalmente ganó la elección.

A lo largo de los cuatro momentos estudiados, quien mantuvo una estrategia de clara restricción en el uso de Facebook fue el candidato López Obrador, quien sólo hizo dos publicaciones., pero las mismas le bastaron para obtener un número casi igual de reacciones, comentarios y compartidos que el candidato Meade Kuribreña, siendo que el segundo hizo muchas más publicaciones. El candidato de la alianza "Juntos haremos historia" optó por utilizar poco la red social en los momentos de mayor exposición para su campaña, haciendo uso de la misma únicamente cuando tenía algo que realmente quería informar o comunicar.

En contraste con este claro uso acotado que hicieron los candidatos de sus perfiles de Facebook, la actividad hecha por los usuarios fue muy copiosa. A partir de los datos generados en el seguimiento, queda claro que la dinámica principal corrió a cargo de quienes 
Rafael Moreno Tostado y José Luis Arriaga Ornelas El acotado uso de Facebook por los candidatos presidenciales en México 2018

reaccionaban a las publicaciones de los candidatos, generando miles de comentarios, reacciones y hasta debates. Hay evidencia (Túñez y Sixto, 2011) de que las características de Internet y sobre todo de la Web 2.0 proporcionan una herramienta potencialmente óptima para generar debate político e interacción con los ciudadanos, pero también se ha documentado (Casero, 2007; Islas, 2006; Ballesteros, 2017) que es más bien escaso el diálogo que se genera en redes como Facebook por parte de los políticos y los ciudadanos.

En una red social como Facebook el usuario se encuentra en un entorno donde puede realizar acciones comunicativas que no son posibles con otros medios, como en los spots de TV o radio, y ni siquiera en los mítines políticos, donde la dinámica comunicativa es distinta. Un usuario puede, por medio de esta red, informarse de lo que hacen los candidatos e interactuar con simpatizantes y detractores de cada uno de ellos. En pocas palabras, existe posibilidad de diálogo, libre expresión y debate en tiempo real. Pero la evidencia demuestra (Cárdenas, Ballesteros y Jara, 2017) que los políticos continúan dudando de la eficacia real de las redes sociales en su aplicación a las campañas electorales, y la comunicación. Y en el caso estudiado, quedó demostrado que para el candidato que resultó ganador, Facebook no fue sino complemento de su estrategia electoral.

A partir de la información obtenida en este trabajo se puede asegurar que redes sociales como Facebook tienen un alto potencial para la difusión de contenidos, no sólo de entretenimiento, sino también en materia de política, ya que pueden llegar a miles o millones de personas a un costo menor al de los medios tradicionales. $Y$ si bien este tipo de plataformas podrían estar reduciendo el poder de los medios tradicionales no hay evidencia de que los resultados electorales estén relacionados con un cambio en la estrategia, apostando por las redes sociales (recuérdese que el candidato López Obrador tuvo menos spots que sus adversarios, pero también menos actividad en Facebook)

No hay ninguna novedad en señalar la potencial capacidad de las redes sociales para generar interlocución política, pero al menos en la más reciente elección presidencial en México ninguno de los candidatos la empleó como herramienta para la comunicación real con los usuarios. Su uso no sólo fue limitado sino que el tipo de publicaciones hechas por los candidatos y sus equipos de campaña fueron mensajes básicamente unidireccionales, sin una apertura intencional encaminada a fomentar el diálogo y la retroalimentación entre los participantes. Es evidente que, sin distinción de colores entre partidos políticos, dicho modelo comunicativo ha sido heredado de generación en generación, como un método para comunicarse con la ciudadanía, sin dar a ésta la ocasión de responder.

En relación a la propuesta de Mancini (1995), sobre las distintas etapas de la comunicación política, a partir de los datos de esta investigación no es posible afirmar la existencia de una 
nueva etapa (que en su caso se caracterizara por el incremento de capacidad comunicativa a través de las redes sociales y una correspondiente disminución del poder de los medios masivos), pero sí hay algunos indicios de que algo se está moviendo: ello queda evidenciado en las interacciones que se detonaron a partir de la emisión de mensajes de los personajes políticos estudiados. Aunque los candidatos no buscaron interactuar con sus seguidores, los seguidores sí interactúan copiosamente entre ellos; debaten, cuestionan, se descalifican, se insultan. Es aquí donde se está pasando algo y debe atenderse, porque hay un inédito volumen de comunicación política entre los usuarios de las redes sociales que quizá (por ahora) no sigue otra agenda que la marcada por los candidatos, pero en cualquier momento podría hacerlo, en la medida que la actividad por parte de los políticos siga siendo del tipo que rehuye a la interacción, como se vio en la campaña bajo estudio.

\section{Bibliografía}

Aceves, F. (2005). Marketing político y rentabilidad electoral: el caso del Distrito 10 de Jalisco en las elecciones federales de 2003. Comunicación y Sociedad, (4), pp. 39-66.

Báez, C. (2002). El Partido Revolucionario Institucional. Los Años Recientes. Convergencia Revista De Ciencias Sociales, (27), pp. 233-283. Recuperado de https://convergencia.uaemex.mx/article/view/1730

Ballesteros, C. A. (2017). Movilización en las campañas electorales a través de las redes sociales. Las elecciones generales españolas de 2015. Conferencia dictada en Cartagena de Indias, Colombia en la International Association for Media and Communication Research. Recuperado de https://www.researchgate.net/publication/320629236 Movilizacion en las campanas electorales a traves de las redes sociales Las elecciones generales espanolas $d$ e 2015

Cárdenas, A., Ballesteros, C. y Jara, R. (2017). Redes sociales y campañas electorales en Iberoamérica. Un análisis comparativo de los casos de España, México y Chile. Cuadernos.info, (41), 19-40. Recuperado de https://doi.org/10.7764/cdi.41.1259

Casero, A. (2007). E-campaña 2007: Internet en las elecciones autonómicas valencianas. Ponencia presentada en el VIII Congreso AECPA 2007, Valencia, pp. 1-18.

Córdova, A. (1989). La Revolución y el Estado en México. México: ERA.

Dader, J. L., Morán, L., Pérez Vizcaíno, R., Tejedor, L., \& Quintana, N. (2017). Qué sabemos y qué podríamos llegar a saber sobre las cibercampañas electorales en España. En J. L. 
Rafael Moreno Tostado y José Luis Arriaga Ornelas El acotado uso de Facebook por los candidatos presidenciales en México 2018

Dader \& E. Campos- Domínguez (Eds.), La búsqueda digital del voto. Cibercampañas Electorales en España 2015-16, Valencia: Tirant Lo Blanch, pp. 435-478.

Dafonte, A. y Martínez, X. (2016). Del view al share: el papel de la comunicación viral en la transformación del ecosistema mediático, Revista Palabra Clave, 19(2), pp. 501-525. Recuperado de: http://www.redalyc.org/articulo.oa?id=64944803007 Consultado el 29 de mayo de 2019.

Delany, C. (2009). Learning from Obama: Lessons for online communicators in 2009 and beyond. Recuperado de http://www.epolitics.com/learning-from-obama/

Eulau, H., Eldersveld, S.J. y Janowitz, M. (eds.) (1956). Political Behavior. Nueva York: Free Press.

Fernández, F. (1996). Los medios de difusión masiva en México. México: Juan Pablos.

Guerrero, M. (2003). ¿Qué es la comunicación política? Ensayo de un modelo. Revista Iberoamericana de Comunicación, (5), pp. 55-86.

(2010). “los medios de comunicación y el régimen político”. En Loeza, So. Y Prud'homme (coord.) Los grandes problemas de México Vol. XIV. México: el Colegio de México, pp. 231-300.

Islas, O. (2006). El Empleo de Internet en el Desarrollo de las Campañas de los Candidatos a la Presidencia de México. Razón y Palabra, (51). Recuperado de http://www.razonypalabra.org.mx/anteriores/n51/oislas.html

Juárez, J. (2007). Las elecciones presidenciales del 2006 a través de los spots de campaña.Espiral (Guadalajara), 14(40), pp. 63-91. Recuperado de http://www.scielo.org.mx/scielo.php?script=sci arttext\&pid=S1665$05652007000100003 \& \operatorname{lng}=\mathrm{es} \&$ tIng $=\mathrm{es}$

Kaid, L. (2004). Handbook of Political Communication Research. New Jersey: Lawrence Erlbaum Associates.

Kuschick, M. (2009). Marketing y comunicación política. El Cotidiano, (155), pp. 31-41.

Kreiss, D. (2015). Digital Campaigning. En Freelon, D. y Coleman, S. (Eds.), Handbook of digital politics. New York: Edward Elgar, pp. 118-135.

Mancini, P. (1995). Americanización y modernización. Breve historia de la campaña electoral, en Comunicación política, Muñoz, A. Y Rospir, J.I. (editores). Editorial Universitas: Madrid.

Mejía, F. (1989). La industria de la radio y la televisión y la política del Estado mexicano, vol.1. México: Fundación Manuel Buendía. 
Rafael Moreno Tostado y José Luis Arriaga Ornelas El acotado uso de Facebook por los candidatos presidenciales en México 2018

Peytibi, F. X., Rodríguez, J. A., y Gutiérrez-Rubí, A. (2008). La experiencia de las elecciones generales del 2008. Revista de Internet, Derecho y Política, (7), 26-37. Recuperado de https://doi.org/10.7238/idp.v0i7.495

Padilla, S. (2018). Marketing político en la elección presidencial mexicana de 2018: las estrategias de AMLO. Econografos Escuela de Administración y Contaduría Pública, (27), pp. 1-43. Recuperado de https://www.researchgate.net/publication/328615961 Marketing politico en la eleccio n presidencial mexicana de 2018 las estrategias de AMLO

Reyes. M. C. (2007). Comunicación política y medios en México: el caso de la reforma a la Ley Federal de Radio y Televisión. Convergencia. Revista de Ciencias Sociales. 14 (43), pp. 105-136.

Rodríguez, R. y Ureña, D. (2012). Diez razones para el uso de Twitter como herramienta en la comunicación política y electoral. Comunicación y Pluralismo, 10, 89-116. Recuperado dehttp://summa.upsa.es/viewer.vm?id=0000030573\&page=1\&search=\&lang=es\&view= main

Trejo Delarbre, R. (1992). La sociedad ausente. Comunicación, democracia y modernidad. México: Cal y Arena.

(2001). Mediocracia sin mediaciones: prensa, televisión y elecciones.

México: Cal y Arena

Tribunal Electoral (2018). Dictamen relativo al cómputo de la elección de Presidente de los Estados Unidos Mexicanos, a la declaración de validez de la elección y a la de presidente electo. Recuperado de https://www.te.gob.mx/Informacion juridiccional/sesion publica/ejecutoria/sentencias/S UP-EEP-0001-2018.pdf

Túñez, M. y Sixto, J. (2011). Redes sociales, política y Compromiso 2.0: La comunicación de los diputados españoles en Facebook. Revista Latina de Comunicación Social, 66, pp. 210-246. Recuperado de https://doi.org/10.4185/RLCS-66-2011-930-210-246

Valdés, M. E. (2015). Comunicación política y propaganda electoral en México. Las reformas de 2007 y 2014. Polis, 11(2), pp. 57-87. Recuperado de http://www.scielo.org.mx/scielo.php?script=sci arttext\&pid=S1870$23332015000200057 \& \operatorname{lng}=\mathrm{es} \& \ln \mathrm{n}=\mathrm{es}$.

Weber, M. (1979). Economía y sociedad. México: Fondo de Cultura Económica.

Zarur, A.E. (1996). El Estado y el modelo de televisión adoptado en México, 1950-2000. México: UAM. 